\title{
Image Retrieval Based on Weighted Block Color Histogram and Texton Co-occurrence Matrix
}

\author{
Wenqing Huang ${ }^{\mathrm{a}}$, Jiazhe Dai ${ }^{\mathrm{b}}$ and Qiang $\mathrm{Wu}^{\mathrm{c}}$ \\ ${ }^{1}$ School of Informatics, Zhejiang Sci-Tech University, Hangzhou 310018,China \\ apatternrecog@163.com, ${ }^{\text {bjz_dai@126.com, }{ }^{c} 1161941191 @ q q . c o m}$
}

\begin{abstract}
Keywords: image retrieval; HSV color histogram; texton co-occurrence matrix; feature extraction. Abstract. Image retrieval is an important topic in the fields of image processing, pattern recognition and computer vision. In this paper, we proposed a new and efficient method of image retreival by combining the color and texture features. We used HSV color histogram for color feature extraction and texton co-occurrence matrix for texture feature extraction. In color feature extraction, the image was divided into nine unequal blocks first and then for each block HSV color histogram was computed. In texture feature extraction, the gray level image was filtered with four predefined textons first and then we used co-occurrence matrix to extract the texture feature of the filtered texton image. After feature extraction, we used Euclidean distance to measure the similarity of the query image and images in the database. We emploied Corel 1000 database for our experiment, the experimental results show that our proposed method performs better than the previous methods.
\end{abstract}

\section{Introduction}

With the rapid development of multimedia and computer vision technology, the increased number of images taken by digital devices has spread across the world. How to search the images that users need in the image database has become one of the main topics in the fields of computer vision and pattern recognition. Generally speaking, the existing image retrieval methods can be primarily splitted into three categories: text-based, content-based and semantic-based. The text-based image retrieval system requires much human labor for annotation. The content based image retrieval system requires people to extract various low-level features such as color, texture and shape from the images. Semantic based image retrieval system uses high-level features to interpret images and measure their similarity. Nowadays, content based image retrieval still attracts much attention by researchers [1].

Color and texture are very pre-attentive to human visual perception and natural scenes are usually rich in both color and texture [2]. In this paper, we combined the color and texture features for image retrieval. There are many color feature descriptors now, such as color histogram, color moments and color correlograms [3]. Among these descriptors, color histogram remains popular due to its simplicity but it lacks spacial information. Wang et al. [4] proposed an improved algorithm of color histogram. He divided the image into several unequal blocks and constructed color histogram of each block. This algorithm performs better than the traditional color histogram. There are also many texture feature descriptors, such as gray co-occurrence matrix and tamura textural feature[3]. In paper [5], Liu et al. proposed the texton co-occurrence matrix (TCM) to describe the textons' correlation for image retrieval. Experimental results show that it has higher accuracy and efficiency than gray co-occurrence matrix. In this paper, we proposed a method based on color histogram of sub-blocks and texton co-occurrence matrix for image retrieval.

The rest of the paper is organized as follows. In section 2, the method of image segmentation and color feature extraction are introduced. Section 3 is the method of texture feature extraction. The similarity measurement is introduced in section 4. Section 5 is the experimental results and section 6 is the conclusion.

\section{Color Feature Extraction}

Image Segmentation. In order to get the information of color spatial distribution, many reserchers have proposed different ways to segment the image, such as in paper [6-8]. In this paper, we divided 
the image into nine unequal blocks according to the proportion 1:2:1 of image length and width. For most images, the importance of the blocks are different and the content is often located in the middle section, so we allocated different weights for them as shown in Fig. 1(c), denote the weights as $\mathrm{w}_{\mathrm{i}}$ $(i=1,2, \ldots, 9)$. We used HSV color histogram to extract the color feature of each block.

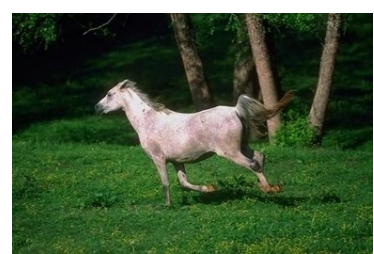

(a)

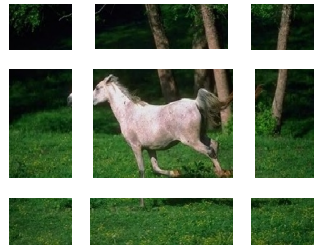

(b)

\begin{tabular}{|l|l|l|}
\hline 1 & 2 & 1 \\
\hline 2 & 3 & 2 \\
\hline 1 & 2 & 1 \\
\hline
\end{tabular}

(c)

Fig. 1 (a) original image (b) segmented image (c) weight of each block

HSV Color Histogram. As HSV color space model is more sensitive to human visual perception than RGB model, we used HSV color space model for color feature extraction in this paper. In order to decrease the dimension of color feature vector, we need to take color quantization. Many researchers have put forward different ways to quantify the color. We used the commonly used method in paper [6] to conduct nonuniform quantization on the three color components. We quantified the $\mathrm{H}$ into 8 pieces, and 3 pieces of $\mathrm{S}$ and $\mathrm{V}$ independently. After quantization, the $\mathrm{H}, \mathrm{S}$ and $\mathrm{V}$ are shown as Eq. 1, Eq. 2, Eq.3 respectively.

$$
\begin{aligned}
& H= \begin{cases}0 & \text { if } h \in(315,20] \\
1 & \text { if } h \in(20,40] \\
2 & \text { if } h \in(40,75] \\
3 & \text { if } h \in(75,155] \\
4 & \text { if } h \in(155,190] \\
5 & \text { if } h \in(190,270] \\
6 & \text { if } h \in(270,295] \\
7 & \text { if } h \in(295,315]\end{cases} \\
& S= \begin{cases}0 & \text { if } s \in(0,0.2] \\
1 & \text { if } s \in(0.2,0.7] . \\
2 & \text { if } s \in(0.7,1]\end{cases} \\
& V= \begin{cases}0 & \text { if } v \in(0,0.2] \\
1 & \text { if } v \in(0.2,0.7] . \\
2 & \text { if } v \in(0.7,1]\end{cases}
\end{aligned}
$$

After color quantization, we combined the three color components into one dimensional feature vector as Eq. 4. $\mathrm{Q}_{\mathrm{s}}$ and $\mathrm{Q}_{\mathrm{v}}$ are the quantization numbers of $S$ and $\mathrm{V}$, both of them are 3, so we can achieve Eq. 5. $\mathrm{L}$ is an integer and the range of value is $[0,1, \mathrm{~L}, 71]$.

$$
\begin{aligned}
& L=H Q_{s} Q_{v}+S Q_{v}+V . \\
& L=9 H+3 S+V .
\end{aligned}
$$




\section{Texture feature extraction}

We used texton co-occurrence matrix for texture feature extraction in this paper. The concept of "texton" was first proposed by Julesz $[9,10]$ and it's very useful in texture analysis. The difference of textons may form various image features so we used textons in our paper. There are many types of textons in images. In this paper, we used types in paper [2] that four special texton types are defined on a $2 \times 2$ grid, denote those four texton types are $T_{1}, T_{2}, T_{3}, T_{4}$ respectively.

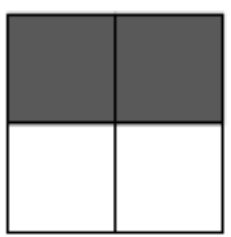

(a)

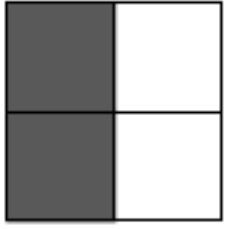

(b)

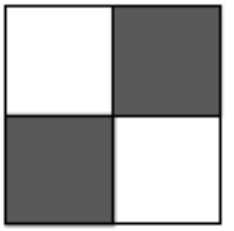

(c)

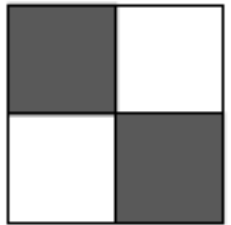

(d)

Fig. 2 Four texton types: (a) $\mathrm{T}_{1}(\mathrm{~b}) \mathrm{T}_{2}(\mathrm{c}) \mathrm{T}_{3}(\mathrm{~d}) \mathrm{T}_{4}$

In order to extract the texture feature of the image, we converted the color image into gray level image first. Then we moved the four texton types from left to right and top to bottom throughout the gray level image with 2 pixels as the step-length to detect textons. If a texton is detected, the original pixels are kept unchanged, otherwise the pixels are set to 0 . After this step, we can obtain four texton images. Then we combined the four texton images together to form the final textons image. After detecting out the final textons image, we need to calculate the emergence number of two value with pixel positions related by $\mathrm{d}$. We used co-occurrence matrixes to extract the feature of texton image. Denote the value of a texton image $\mathrm{f}(\mathrm{x}, \mathrm{y})$ at point $\mathrm{P}(\mathrm{x}, \mathrm{y})$ is $\mathrm{w}, \mathrm{w} \in\{0,1, \ldots, \mathrm{W}-1\}$. Suppose there are two pixels $\mathrm{P}_{1}\left(\mathrm{x}_{1}, \mathrm{y}_{1}\right)$ and $\mathrm{P}_{2}\left(\mathrm{x}_{2}, \mathrm{y}_{2}\right)$, the value of them are $\mathrm{f}\left(\mathrm{P}_{1}\right)=\mathrm{w}_{1}$ and $\mathrm{f}\left(\mathrm{P}_{2}\right)=\mathrm{w}_{2}$. If the probability of two values $\mathrm{w}_{1}$ and $\mathrm{w}_{2}$ co-occurring with two pixel positions related by $\mathrm{d}$ is $\mathrm{P}_{\mathrm{r}}$, we can define the cell entry $\left(\mathrm{w}_{1}, \mathrm{w}_{2}\right)$ of co-occurrence matrix $\mathrm{C}_{\mathrm{d}, \theta}$ :

$$
C_{d, \theta}\left(w_{1}, w_{2}\right)=1-P_{r}\left\{f\left(P_{1}\right)=w_{1} \wedge f\left(P_{2}\right)=w_{2}|| P_{1}-P_{2} \mid=d\right\} .
$$

The value range of $\theta$ is $\left[0^{\circ}, 45^{\circ}, 90^{\circ}, 135^{\circ}\right]$. We used energy, contrast, entropy and homogeneity to discribe the image features. The features are given as follows:

$$
\begin{aligned}
& T_{1}=\sum_{x=0}^{N-1} \sum_{y=0}^{N-1} C_{d, \theta}^{2}(x, y) . \\
& T_{2}=\sum_{x=0}^{N-1} \sum_{y=0}^{N-1}(x-y)^{2} C_{d, \theta}(x, y) . \\
& T_{3}=-\sum_{x=0}^{N-1} \sum_{y=0}^{N-1} C_{d, \theta}(x, y) \log C_{d, \theta}(x, y) . \\
& T_{4}=\frac{\sum_{x=0}^{N-1} \sum_{y=0}^{N-1} C_{d, \theta}(x, y)}{1+(x-y)^{2}} .
\end{aligned}
$$

\section{Similarity Measurement}

Assuming the feature vector of query image is $\mathrm{Q}=\left[\mathrm{Q}_{1}, \mathrm{Q}_{2}, \ldots, \mathrm{Q}_{\mathrm{N}}\right]$ and image in the database is $\mathrm{I}=\left[\mathrm{I}_{1}, \mathrm{I}_{2}, \ldots, \mathrm{I}_{\mathrm{N}}\right]$, the distance of color feature and texture feature are $\operatorname{dis}(\mathrm{Q}, \mathrm{I})_{\mathrm{HIS}}$ and $\operatorname{dis}(\mathrm{Q}, \mathrm{I})_{\mathrm{TCM}}$ respectively. We used Euclidean diatance to calculate dis $(\mathrm{Q}, \mathrm{I})_{\mathrm{HIS}}$ and $\operatorname{dis}(\mathrm{Q}, \mathrm{I})_{\mathrm{TCM}}$. The Euclidean distance is shown as Eq. 11. 


$$
\text { Euclidean distance }=\sqrt{\sum_{i=1}^{N}\left(I_{i}-Q_{i}\right)^{2}} .
$$

As the image was segmented into nine blocks in the process of color feature extraction, we need to calculate the Euclidean distance of each block of query image and the corresponding block of image in the database first. Denote these distances are $d_{i}(1=1,2, \ldots, 9)$, then dis $(Q, I)_{\text {HIS }}$ can be represented as Eq. 12 according to the weight of each block. The similarity of query image and image in the database can be represented as the Eq. 13. In Eq. 13, $\mathrm{p}_{1}$ and $\mathrm{p}_{2}$ are the weights of color and texture feature. The values of them belong to $[0,1]$ and $\mathrm{p}_{1}+\mathrm{p}_{2}=1$. In our experiments, we set $\mathrm{p}_{1}=\mathrm{p}_{2}=0.5$.

$$
\begin{aligned}
& \operatorname{dis}(Q, I)_{H I S}=\sum_{i=1}^{9} w_{i} d_{i} . \\
& \operatorname{dis}(Q, I)=p_{1} \operatorname{dis}(Q, I)_{H I S}+p_{2} \operatorname{dis}(Q, I)_{T C M} .
\end{aligned}
$$

\section{Experimental results}

The Corel 1000 database was used for evaluation of our proposed method and other existing methods. In this paper, we used the precision measure to evaluate the performance of retrieval systems.

$$
\text { Precision }=\frac{\text { Number of relevent images retrieved }}{\text { Total number of images retrieved }} \text {. }
$$

To compare the precision, 20 random experiments were carried out. Fig. 3 is the results of different retrieval systems. The average precision of proposed method and other retrieval systems is shown in Table 1. Fig. 4 shows the comparison of average precision obtained by different retrieval methods.

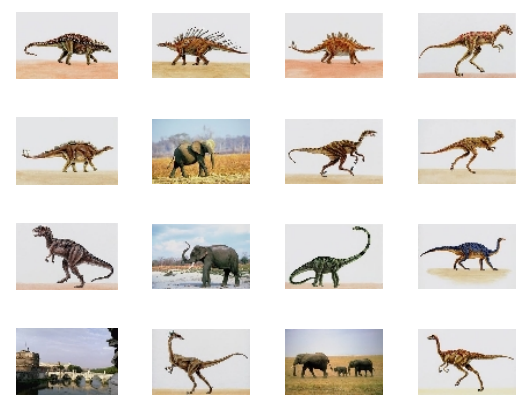

(a)

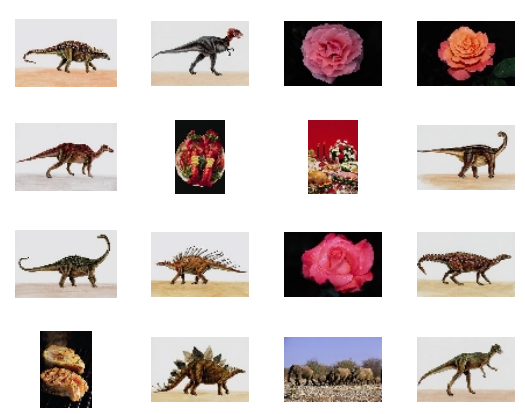

(c)

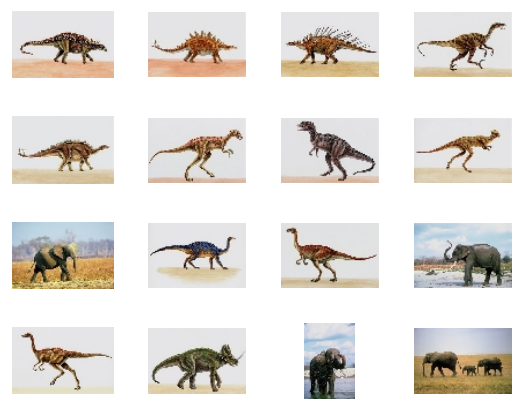

(b)

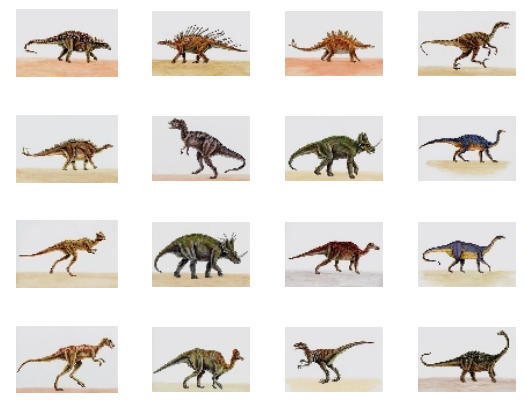

(d)

Fig. 3 Different image retrieval results for the same query image( image 459.jpg in the dataset): (a) result based on HSV color histogram (b) result based on weighted block HSV color histogram (c) result based on TCM texture (d) result based on our proposed method 
Table 1 Average precision

\begin{tabular}{|c|c|c|c|c|}
\hline \multirow{2}{*}{ Class } & \multicolumn{4}{|c|}{ Average Precision using } \\
\cline { 2 - 5 } & $\begin{array}{c}\text { HSV color } \\
\text { histogram }\end{array}$ & $\begin{array}{c}\text { Weighted block } \\
\text { HSV color } \\
\text { histogram }\end{array}$ & $\begin{array}{c}\text { TCM } \\
\text { texture }\end{array}$ & Proposed method \\
\hline Africa & 0.68 & 0.69 & 0.31 & 0.71 \\
\hline Beach & 0.43 & 0.45 & 0.34 & 0.48 \\
\hline Building & 0.50 & 0.55 & 0.32 & 0.63 \\
\hline Bus & 0.67 & 0.72 & 0.40 & 0.87 \\
\hline Dinosaur & 0.97 & 0.98 & 0.91 & 0.99 \\
\hline Elephant & 0.46 & 0.44 & 0.22 & 0.56 \\
\hline Flower & 0.71 & 0.78 & 0.51 & 0.84 \\
\hline Horse & 0.96 & 0.96 & 0.43 & 0.95 \\
\hline Mountain & 0.47 & 0.51 & 0.26 & 0.53 \\
\hline food & 0.60 & 0.72 & 0.31 & 0.73 \\
\hline
\end{tabular}

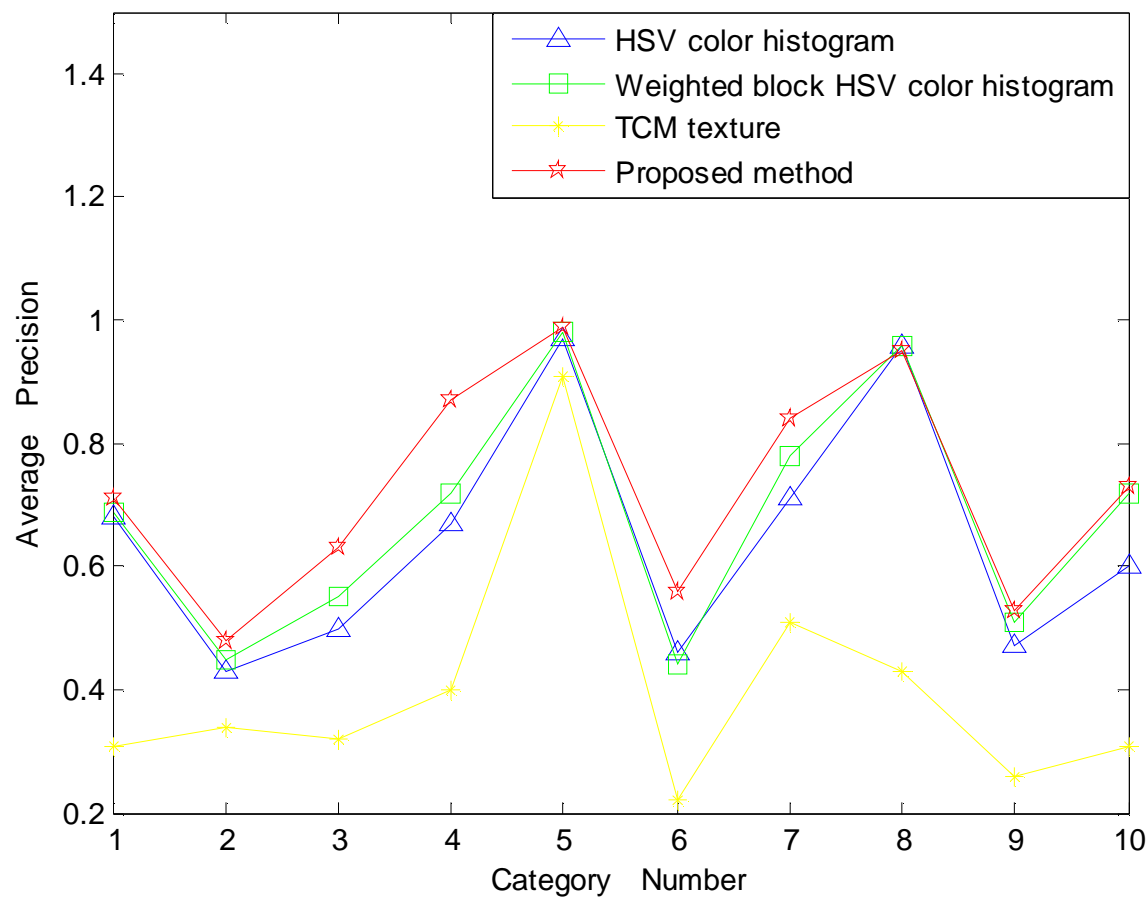

Fig. 4 Precision comparision

\section{Conclusion}

Image retrieval has become an active and important research topic in multimedia information processing. In this paper, we proposed an efficient image retrieval method based on HSV color histogram and texton co-occurrence matrix. As only color feature or only texture feature are not sufficient to describe an image, we combined the two together in this paper. To improve the discriminating power of color feature, we divided the image into nine unequal blocks and allocated different weights for them. The retrieval performance was compared with the HSV color histogram and texton co-occurrence matrix. The experimental results show that the method we proposed performs better than the previous methods.

\section{References}

[1] Datta R, Joshi D, Li J, et al. Image retrieval: Ideas, influences, and trends of the new age. ACM 
Computing Surveys (CSUR), 2008, 40(2): 5.

[2] Liu G H, Zhang L, Hou Y K, et al. Image retrieval based on multi-texton histogram. Pattern Recognition, 2010, 43(7): 2380-2389.

[3] Manjunath B S, Ohm J R, Vasudevan V V, et al. Color and texture descriptors. Circuits and Systems for Video Technology, IEEE Transactions on, 2001, 11(6): 703-715.

[4] Baoping Wang, Jing Zhao, Jiankang Su, et al. Research on local color-space characteristics image retrieval method. Computer application and software, 2012, 29(5):28-31."In Chinese"

[5] Liu G H, Yang J Y. Image retrieval based on the texton co-occurrence matrix. Pattern Recognition, 2008, 41(12): 3521-3527.

[6] Kavitha C, Rao D B P, Govardhan D A. Image retrieval based on color and texture features of the image sub-blocks. International Journal of Computer Applications (0975-8887), 2011, 15(7).

[7] Tamilkodi R, Karthika R A, RoslineNesaKumari G, et al. Segment Based Image Retrieval Using HSV Color Space and Moment. Emerging ICT for Bridging the Future-Proceedings of the 49th Annual Convention of the Computer Society of India (CSI) Volume 1. Springer International Publishing, 2015: 239-247.

[8] Sajwan V, Goyal P. Sub-block Features Based Image Retrieval. Computational Intelligence in Data Mining-Volume 1. Springer India, 2015: 637-646.

[9] Bela Julesz, Textons, the elements of texture perception, and their interactions, Nature, 1981.

[10] Bela Julesz, Texton gradients: The texton theory revisited, Biological Cybernetics, vol. 54, no. 4-5, pp. 245-251, 1986. 